\title{
Study on identification method of railway dynamic bottleneck station under abnormal conditions
}

\author{
Zihan $\mathrm{Qu}^{1}$ and Shiwei $\mathrm{He}^{2}{ }^{2 *}$ \\ ${ }^{1}$ Key Laboratory of Transport Industry of Big Data Application Technologies for Comprehensive \\ Transport, Ministry of Transport, Beijing Jiaotong University, Beijing, Beijing, 100044, \\ China;1343907389@qq.com \\ 2 School of Transportation, Beijing Jiaotong University, Beijing, Beijing, 100044, China; \\ shwhe@bjtu.edu.cn \\ * Corresponding author's e-mail :shwhe@bjtu.edu.cn
}

\begin{abstract}
In this paper, the physical station of space network was extended in time dimension by combining the train diagram information and station technical operation standard time. At the same time, the topology of railway space-time network which considered the secondary operation process of train was constructed and an improved $\mathrm{A}^{*}$ algorithm based on car flow routing was proposed to generate feasible path sets. On this basis, a dynamic car flow organization optimization model was built to simulate the railway car flow organization process under abnormal conditions, and the results of solving the model could be used to obtain the real-time quantity of cars at each station.This paper can identify the dynamic bottleneck by comparing the real-time quantity of cars with the maximum quantity of cars at the station. Finally, the feasibility of this method was analyzed and verified by a case.
\end{abstract}

Key words: railway transportation; time-space network; dynamic bottleneck; car flow organization

\section{Introduction}

When the line is interrupted or the station stops operation due to natural disasters and other abnormal conditions, the operation of relevant trains will be affected. After a period of time, the car flow in some stations will be overstocked, thus forming a bottleneck. Reasonable estimation of dynamic bottleneck station will be helpful for railway departments to take car flow adjustment measures in time. In terms of traffic bottleneck research, there are many researches on dynamic bottleneck of road [1-5] and subway [6-9], but few researches on dynamic bottleneck of railway, and there is a lack of research on identification method of railway dynamic bottleneck station under abnormal condition. The core problem of dynamic bottleneck station identification is car flow estimation, which is closely related to dynamic car flow organization. Traditional car flow organization model such as empty car allocation model [11] is aimed at static car flow, and lacks consideration of secondary operation of loaded car flow. The innovation points of this paper are as follows: Based on the train diagram, the time-space network was constructed for the first time by considering the secondary operation of loaded car flow and other local operation processes. For the first time, an improved $\mathrm{A}^{*}$ algorithm based on car flow routing was used to generate a feasible path set of any pair of transportation demands. For the first time, an optimization model of dynamic car flow organization under abnormal conditions was established to simulate the process of railway car flow organization under abnormal conditions, and the car flow distribution in the future period was estimated based on the results of car flow assigning in the space-time 
network, so as to estimate the station with bottleneck and the period when the station is at a bottleneck.

\section{Methods}

\subsection{Construction of space-time network based on the train diagram}

In order to simplify the scale of the problem, a railway bureau is taken as the research object, and the marshalling station, district station, large freight station and boundary station of the railway bureau are taken as the main fulcrum stations, and the car flow of the intermediate station is merged in accordance with the section center rule [12]. Set the fulcrum station set in the railway bureau as $\mathrm{S}$, and define the space-time network as $G=(N, A)$, where $\mathrm{N}$ is the set of nodes in the network and $\mathrm{A}$ is the set of arcs in the network. The network takes 18:00 of the current day to 18:00 of the next day as a decision cycle. The train diagram is the main frame of constructing space-time network.

Network $G$ takes the arrival node (AN) and departure node (DN) of the train path as the main space-time nodes. The loaded car state end node (LCSEN) and the empty car state end node (ECSEN) represent the mutual transformation of loaded car flow and empty car flow. Secondary operation completion node (SOCN) represents the completion time of secondary operation of loaded car flow. Each station sets two initial time nodes (ITN), which are divided into loaded car layer and empty car layer, to represent the beginning of the car flow in the decision cycle. Each station sets two end time nodes (ETN), which are also divided into loaded car layer and empty car layer, to represent the end of the car flow in the decision cycle. The type Numbers of space-time nodes are shown in table 1.

Table 1. Type Numbers of space-time nodes

\begin{tabular}{cc}
\hline Space-time node type & Type number \\
\hline AN & 1 \\
DN & 2 \\
LCSEN & 3 \\
ECSEN & 4 \\
SOCN & 5 \\
ITN & 6 \\
ETN & 7 \\
\hline
\end{tabular}

Network $G$ takes the train path as the train arc (TA), and adds corresponding local operation arc (LOA) and secondary operation arc (SOA) based on the technical operation time and cargo operation time of each fulcrum station, and realizes the connection of each arc through marshalling and departing arc (MADA), empety car allocation arc (ECAA), transfer operation arc (TOA) and stay arc (SA). The initial arc (IA) represents the initialization of the car flow. The end arc (EA) and the detention arc (DA) represent the process of car flow detention to the end of the decision cycle. At the same time, hierarchy attributes were added to distinguish loaded car layer (LCL), empty car layer (ECL) and public layer (PL). At present, the research on dynamic car flow organization lacks the consideration of secondary operation of loaded car flow, and this paper represents the secondary operation process of loaded car flow through the connection between the LCSEN and SOCN. The network topology relationship between space-time nodes and space-time arcs is shown in figure 1 . The type 
Numbers of space-time arcs are shown in table 2 and the hierarchy numbers of Hierarchy set are shown in table 3.

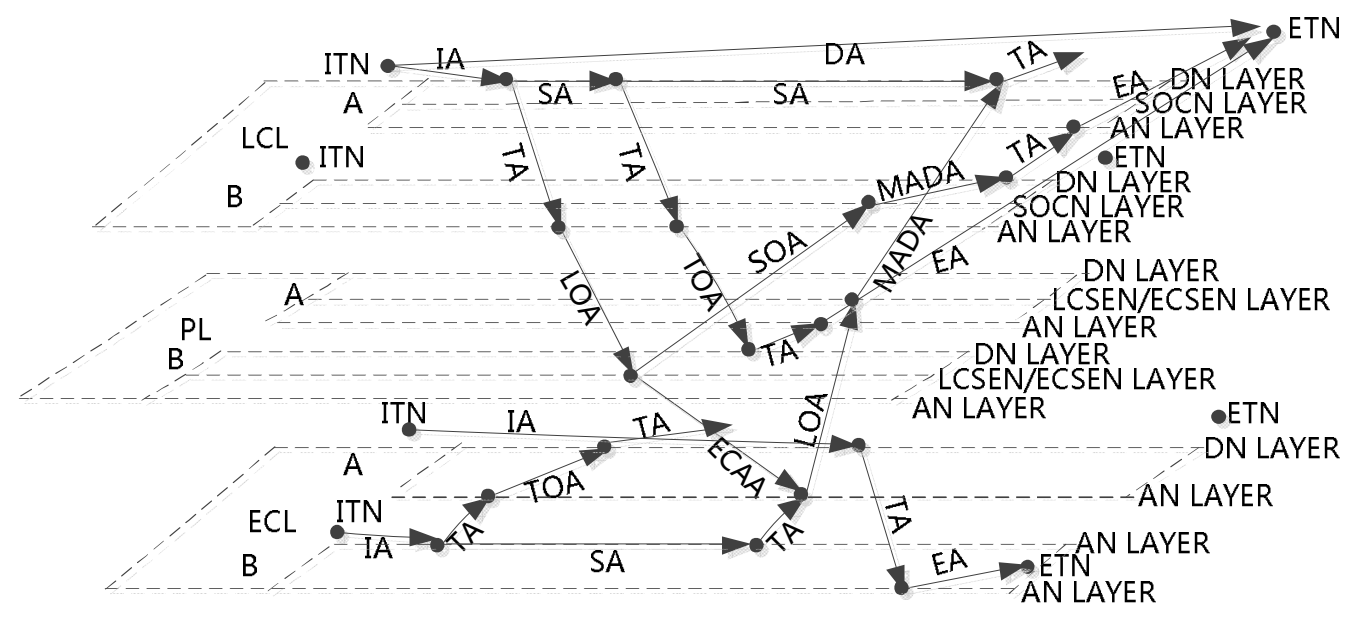

Figure 1. Schematic diagram of space-time network

Table 2. Type Numbers of space-time arcs

\begin{tabular}{cc}
\hline Space-time arc type & Type number \\
\hline TA & 1 \\
LOA & 2 \\
SOA & 3 \\
MADA & 4 \\
ECAA & 5 \\
TOA & 6 \\
SA & 7 \\
IA & 8 \\
EA & 9 \\
DA & 10 \\
\hline
\end{tabular}

Table 3. Hierarchy numbers of hierarchy

\begin{tabular}{cc}
\hline Hierarchy & Hierarchy number \\
\hline LCL & 1 \\
ECL & 2 \\
PL & 3 \\
\hline
\end{tabular}

Because loaded car flow has the characteristics of tree path[13-14], this paper describes the transport process of loaded car flow by constructing space-time path which is a set of space-time arcs that satisfy the constraint of time continuity. The space-time path of loaded car flow is divided into four categories: initial path (IP), secondary operation path (SOP), empty to loaded path (ETLP) and detention path (DP). The IP represents the transport process of the initial loaded car flow. The path starts from the ITN and ends at the LCSEN. The path takes the LOA as the final arc.The SOP represents the transport process of secondary operation loaded car flow. The path starts from the LCSEN and ends at the ETN. The path takes the SOA 
as the first arc and cannot contain the LOA. ETLP represents the transport process of self-loading car flow. The path starts from the ECSEN and ends at the ETN. The DP represents the loaded car flow remains at the station throughout the decision cycle. The path starts from the ITN and ends at the ETN. The type Numbers of space-time paths are shown in table 4.

Table 4. Type Numbers of space-time paths

\begin{tabular}{cc}
\hline Space-time path type & Type number \\
\hline IP & 1 \\
SOP & 2 \\
ETLP & 3 \\
DP & 4 \\
\hline
\end{tabular}

\subsection{Feasible path set generation algorithm based on improved $A^{*}$ algorithm}

According to the requirements of train marshalling plan, any pair of OD demands has a fixed car flow connection mode, thus each pair of OD demands has specific routing, that is, the car flow routing. In this paper, the feasible path has a broader meaning in the space-time network. For any pair of OD demands, a set of space-time arc sets that satisfy the time continuity constraint and the constraint of the car flow routing station sequence is a feasible space-time path. Set OD demand number as $\mathrm{f}$, and the algorithm for generating feasible path set is as follows:

Step1: Take $\mathrm{f}=1$, and turn Step2;

Step2: Set the maximum number of K-shortest path of demand $f$ to $\mathrm{N}$, and the initial $\mathrm{K}=0$, set the starting station of demand $\mathrm{f}$ as Station1, and the final station as Station2. Traverse the car flow routing set, find the car flow routing of starting station as Station1 and ending station as Station2, and record the station sequence corresponding to the car flow routing as stationList, and turn Step3;

Step3: Taking the ETN corresponding to the Station2 as the source node, Dijkstra algorithm is used to find the shortest distance between the source node and other nodes in the space-time network, which is taken as the $\mathrm{H}$ value of each node, and turn Step4;

Step4: Establish a node set as closeList which have been estimated and a node set as openList which will be estimated. Add the ITN corresponding to the Station1 to openList, and turn Step5;

Step5: Traverse the nodes in openList to find the node with the lowest $\mathrm{F}$ value and treat it as $\mathrm{m}$ to be processed, and turn Step6;

Step6: Move this node from openList to closeList, and turn Step7;

Step7: For each pointing node of $\mathrm{m}$ as $\mathrm{n}$, set its parent node as $\mathrm{m}$, and recalculate its $\mathrm{G}$ and $\mathrm{F}$ values, and add them to openList, and turn Step8;

Step8: If openList is empty, the target $\mathrm{K}$ short path is not found, and the search fails, then $\mathrm{f}=\mathrm{f}+1$, and turn Step2.If openList is not empty, traverse openList, if the node with the lowest $\mathrm{F}$ value is the ETN corresponding to the Station2, the target $\mathrm{K}$ shortest path is found, then $\mathrm{K}=\mathrm{K}+1$, and turn Step9; otherwise turn Step5;

Step9: First, judge whether $\mathrm{K}$ is greater than $\mathrm{N}$, if $\mathrm{K}$ is greater than $\mathrm{N}, \mathrm{f}=\mathrm{f}+1$, then turn Step2.Otherwise, judge the number of LOA in the K shortest path. If the number is greater than 1, remove the K short path and turn Step4; if the number is equal to 1, divide the $\mathrm{K}$ short path into two paths with the end node of the LOA as the cutting node, and turn Step10; 
Step10: Take the station sequence of these two paths as stationList1 and stationList2. Determines whether the station sequence of each path is the same as stationList, and if so, adds the path to the feasible path set, and turn Step5;

Step11: Until all OD demands are traversed to generate a feasible path set, the algorithm is finished.

\subsection{Model of dynamic car flow organization optimization under abnormal conditions}

At present, there is a blank research on the identification of railway dynamic bottleneck stations under abnormal conditions in China. The key to identify the dynamic bottleneck station is to grasp the real-time dynamic change of the number of cars stored in the station. The train runs according to the train diagram, and the space-time network constructed based on the train diagram conforms to the situation of railway field operation. In this paper, based on the space-time network, the distribution results of car flow on the space-time network are obtained by establishing an optimization model of dynamic car flow organization under abnormal conditions. According to the arc flow, the number of cars saved at any time at each station can be obtained. By comparing the number of cars saved in real time with the maximum number of cars in the station, it can be concluded whether the station belongs to the bottleneck stage at any moment, so as to realize the identification of dynamic bottleneck station.

\subsubsection{Sets parameter and variable definitions}

The set parameters of the model are defined as follows:

$H--$ Hierarchy number set;

$N--$ Space-time node set;

$B_{N}--$ Set of type numbers of space-time nodes;

$b_{n}$ - - Type number of space-time node $n, b_{a} \in B_{A}, n \in N$;

$h_{n}--$ Hierarchy number of space-time node, $h \in H, n \in N$;

$A$ - - Space-time arc set;

$A_{n s}-$-Set of space-time arcs starting from space-time node $n, n \in N$;

$A_{n \mathrm{e}}--$ Set of space-time arcs ending in space-time node $n, n \in N$;

$B_{A}--$ Set of type numbers of space-time arcs;

$b_{a}-$-Type number of space-time $\operatorname{arc} a, b_{a} \in B_{A}, a \in A$;

$h_{a}--$ Hierarchy number of space-time $\operatorname{arc} a, h \in H, a \in A$;

$t_{a}--$ Time of space-time arc $a, a \in A$;

$c_{a}--$ Capacity of space-time $\operatorname{arc} a, a \in A$;

$P--$ Space-time path set;

$P_{n s}--$ Set of space-time paths starting from space-time node $n, n \in N$;

$P_{n e}--$ Set of space-time paths ending in space-time node $n, n \in N$;

$B_{P}--$ Set of type numbers of space-time paths;

$b_{p}--$ Type number of space-time path $p, b_{p} \in B_{P}, p \in P$;

$h_{p}--$ Hierarchy number of space-time path $p, h \in H, p \in P$;

$t_{p}--$ Time of space-time path $p, p \in P$;

$A_{p}--$ Space-time arc set of space-time path $p, p \in P$;

$F_{1}$ - - Initial loaded car flow set;

$P_{f 1}--$ Set of feasible paths of initial loaded car flow $f 1, f_{1} \in F_{1}$;

$q_{f 1}--$ Amount of initial loaded car flow $f 1, f_{1} \in F_{1}$; 
$F_{2}-$ - Initial empty car flow set;

$A_{f 2}--$ Set of feasible arcs of initial empty car flow $f 2, f_{2} \in F_{2}$;

$q_{f 2}--$ Amount of initial empty car flow $f 2, f_{2} \in F_{2}$;

$L--$ Loading plan set;

$q_{l}--$ Planned loading quantity for one day of loading plan $l, l \in L$;

$P_{l}--$ Space-time path set in line with loading plan $l, l \in L$;

$r_{l}--$ Completed loading quantity of loading plan $l$ when abnormal conditions occur, $l \in L$;

$E$ - - Emptying plan set;

$q_{e}--$ Planned emptying quantity for one day of emptying plan $e, e \in E$;

$A_{e}--$ Space-time arc set in line with emptying plan $e, e \in E$;

$r_{e}--$ Completed emptying quantity of emptying plan $e$ when abnormal conditions occur, $e \in E$;

$m--$ Maximum number of marshalling cars in a train;

$A_{T}--$ Train arc set affected under abnormal conditions.

The main decision variables of the model are as follows:

$x_{a}--$ Empty car flow quantity of space-time $\operatorname{arc} a$;

$y_{p}-$-Loaded car flow quantity of space-time path $p$.

The auxiliary decision variables of the model are as follows:

$z_{p}^{f 1}--$ Loaded car flow quantity of feasible path $p$ of initial loaded car flow $f 1$;

$v_{a}^{f 2}--$ Empty car flow quantity of feasible arc $a$ of initial empty car flow $f 2$.

\subsubsection{Objective function}

The objective is to compress the transit time of loaded cars at the station, reduce the running time of empty cars and minimize the quantity of off-axle cars. The expression is as follows:

$$
\min Z=\sum_{p \in P}\left(t_{p}-\sum_{a \in A_{p} \mid\left(b_{a}=1\right)} t_{a}\right) \times y_{p}+\sum_{\left.a \in A \mid h_{a} \neq 1, b_{a} \neq 7, b_{a} \neq 10\right)} t_{a} \times x_{a}+\sum_{a \in A \mid b_{a}=1}\left(m-\sum_{p \in P \mid a \in A_{p}} y_{p}-x_{a}\right)
$$

\subsubsection{Constraint condition}

Initial loaded car flow constraints: For each loaded car flow, the sum of the flows allocated on the feasible path is equal to the quantity of initial loaded car flow.

$$
\sum_{p \in P_{f 1}} z_{p}^{f 1}=q_{f 1}, \quad \forall f_{1} \in F_{1}
$$

Initial empty car flow constraints: For each empty car flow, the sum of the quantity of flows allocated on the feasible arc is equal to the quantity of initial empty car flow.

$$
\sum_{a \in A_{f 2}} v_{a}^{f 2}=q_{f 2}, \quad \forall f_{2} \in F_{2}
$$

Consistent constraints of decision variables: For each IP or DP, the sum of the quantity of initial loaded car flows allocated on that path is equal to the the quantity of flows of that path; 
for each IA or DA, the sum of the quantity of initial empty car flows allocated on that arc is equal to the the quantity of flows of that arc.

$$
\begin{gathered}
\sum_{f_{1} \in F_{1} \mid p \in P_{f_{1}}} z_{p}^{f_{1}}=y_{p}, \quad \forall p \in P \mid\left(b_{p}=1 \text { or } b_{p}=4\right) \\
\sum_{f_{2} \in F_{2} \mid a \in A_{f_{2}}} v_{a}^{f_{2}}=x_{a}, \quad \forall a \in A \mid\left(\left(b_{a}=8 \text { or } b_{a}=10\right), h_{a}=2\right)
\end{gathered}
$$

Space-time arc capability constraints: For each space-time arc, the sum of the quantity of loaded car flows on that arc and the quantity of empty car flows on that arc cannot exceed the capacity of that arc.

$$
\sum_{p \in P \mid a \in A_{p}} y_{p}+x_{a} \leq c_{a}, \quad \forall a \in A
$$

Consistent constraints of exchange of LCL and ECL For each LCSEN, the loaded car flow quantity of the IP ending at that node is equal to the sum of the loaded car flow quantity of the SOP starting from that node and the empty car flow quantity of the ECAA starting from that node; For each ECSEN, the empty car flow quantity of the LOA ending at that node is equal to the sum of the loaded car flow quantity of the ETLP starting from that node.

$$
\begin{gathered}
\sum_{p \in P_{n e} \mid b_{p}=1} y_{p}=\sum_{p \in P_{n s} \mid b_{p}=2} y_{p}+\sum_{a \in A_{n s} \mid b_{a}=5} x_{a}, \quad \forall n \in N \mid b_{n}=3 \\
\sum_{a \in A_{n e} \mid\left(b_{a}=2 \text { or } b_{a}=8\right)} x_{a}=\sum_{p \in P_{n s} \mid b_{p}=3} y_{p}, \quad \forall n \in N \mid b_{n}=4
\end{gathered}
$$

Flow conservation constraints of node in ECL:For each space-time node in ECL or PL, the sum of the empty car flow quantity of the space-time arc ending at that node in ECL or PL is equal to the sum of the empty car flow quantity of the space-time arc starting from that node in ECL or PL.

$$
\sum_{a \in A_{n e} \mid h_{a} \neq 1} x_{a}=\sum_{a \in A_{n s} \mid h_{a} \neq 1} x_{a}, \quad \forall n \in N \mid h_{n} \neq 1
$$

Loading plan constraints: For each loading plan, the sum of the loaded car flow quantity of the space-time paths in line with the loading plan cannot exceed the planned loading quantity for one day of the plan, nor can it be lower than completed loading quantity of the plan when abnormal conditions occur.

$$
r_{l} \leq \sum_{p \in P_{l}} y_{p} \leq q_{l}, \quad \forall l \in L
$$

Emptying plan constraints: For each emptying plan, the sum of the empty car flow quantity of space-time arcs in line with the emptying plan cannot exceed the planned emptying quantity for one day of the plan, nor can it be lower than completed emptying quantity of the plan when abnormal conditions occur.

$$
r_{\mathrm{e}} \leq \sum_{a \in A_{e}} x_{a} \leq q_{e}, \quad \forall e \in E
$$

Train operation restrictions constraints under abnormal conditions: For each affected TA, the sum of the quantity of loaded car flow on that arc and the quantity of empty car flow on that arc is equal to zero. 


$$
\sum_{p \in P \mid a \in A_{p}} y_{p}+x_{a}=0, \quad \forall a \in A_{T}
$$

\subsubsection{Model synthesis}

By combining constraints (1) to (12), the model of dynamic car flow organization optimization under abnormal conditions can be obtained.

MDCFOOAC :

$$
\min Z=\sum_{p \in P}\left(t_{p}-\sum_{a \in A_{p} \mid\left(b_{a}=1\right)} t_{a}\right) \times y_{p}+\sum_{a \in A \mid\left(h_{a} \neq 1, b_{a} \neq 7, b_{a} \neq 10\right)} t_{a} \times x_{a}+\sum_{a \in A \mid b_{a}=1}\left(m-\sum_{p \in P \mid a \in A_{p}} y_{p}-x_{a}\right)
$$

s.t.

$$
\begin{gathered}
\sum_{p \in P_{f 1}} z_{p}^{f 1}=q_{f 1}, \quad \forall f_{1} \in F_{1} \\
\sum_{a \in A_{f 2}} v_{a}^{f 2}=q_{f 2}, \quad \forall f_{2} \in F_{2} \\
\sum_{f_{1} \in F_{1} \mid p \in P_{f 1}} z_{p}^{f_{1}}=y_{p}, \quad \forall p \in P \mid\left(b_{p}=1 \text { or } b_{p}=4\right) \\
\sum_{f_{2} \in F_{2} \mid a \in A_{f 2}} v_{a}^{f_{2}}=x_{a}, \quad \forall a \in A \mid\left(\left(b_{a}=8 \text { or } b_{a}=10\right), h_{a}=2\right) \\
\sum_{p \in P_{n e} \mid b_{p}=1} y_{p}=\sum_{p \in P_{n s} \mid b_{p}=2} y_{p}+\sum_{a \in A_{n s} \mid b_{a}=5} \quad x_{a}, \quad \forall n \in N \mid b_{n}=3 \\
\sum_{a \in A_{n e} \mid\left(b_{a}=2\right.} y_{o r} x_{\left.b_{a}=8\right)}=\sum_{p \in P_{n s} \mid b_{p}=3} y_{p}, \quad \forall n \in N \mid b_{n}=4 \\
r_{l} \leq \sum_{p \in P_{l}} y_{p} \leq q_{l}, \quad \forall l \in L \\
r_{\mathrm{e}} \leq \sum_{a \in A_{e}} x_{a} \leq q_{e}, \quad \forall e \in E \\
\sum_{p \in P \mid a \in A_{p}} y_{p}+x_{a}=0, \quad \forall a \in A_{T}
\end{gathered}
$$
follows:

Change constraint (10) and constraint (11) into constraint (13) and constraint (14) as

$$
\begin{array}{ll}
\sum_{p \in P_{l}} y_{p} \leq q_{l}, & \forall l \in L \\
\sum_{a \in A_{e}} x_{a} \leq q_{e}, & \forall e \in E
\end{array}
$$

Combining constraints (1) to (9), together with constraint (13) and constraint (14), the model of dynamic car flow organization optimization under normal conditions can be obtained.

MDCFOONC :

$$
\min Z=\sum_{p \in P}\left(t_{p}-\sum_{a \in A_{p} \mid\left(b_{a}=1\right)} t_{a}\right) \times y_{p}+\sum_{a \in A \mid\left(h_{a} \neq 1, b_{a} \neq 7, b_{a} \neq 10\right)} t_{a} \times x_{a}+\sum_{a \in A \mid b_{a}=1}\left(m-\sum_{p \in P \mid a \in A_{p}} y_{p}-x_{a}\right)
$$

s.t. 


$$
\begin{gathered}
\sum_{p \in P_{f 1}} z_{p}^{f 1}=q_{f 1}, \quad \forall f_{1} \in F_{1} \\
\sum_{a \in A_{f 2}} v_{a}^{f 2}=q_{f 2}, \quad \forall f_{2} \in F_{2} \\
\sum_{f_{1} \in F_{1} \mid p \in P_{f 1}} z_{p}^{f_{1}}=y_{p}, \quad \forall p \in P \mid\left(b_{p}=1 \text { or } b_{p}=4\right) \\
\sum_{f_{2} \in F_{2} \mid a \in A_{f 2}} v_{a}^{f_{2}}=x_{a}, \quad \forall a \in A \mid\left(\left(b_{a}=8 \text { or } b_{a}=10\right), h_{a}=2\right) \\
\sum_{p \in P_{n e} \mid b_{p}=1} \sum_{p \in P \mid a \in A_{p}} y_{p}+x_{a} \leq c_{a}, \quad \forall a \in A \\
\sum_{a \in A_{n e} \mid\left(b_{a}=2\right.} y_{\text {or }} y_{\left.b_{a}=8\right)} y_{p}=2 \sum_{a \in A_{n s} \mid b_{a}=5} x_{a}, \quad \forall n \in N \mid b_{n}=3 \\
\sum_{p \in P_{l}} y_{p} \leq q_{l} \mid b_{p}=3 \\
\sum_{a \in A_{e}} x_{a} \leq q_{e}, \quad \forall n \in N \mid b_{n}=4
\end{gathered}
$$

\section{Case study}

Set up a small network, where A, B, C and D are fulcrum stations and D is the boundary station. Set the arrival time, disintegration time, assembly time, grouping time and departure time of each fulcrum station as $30 \mathrm{~min}$, loading time and unloading time of each fulcrum station as $180 \mathrm{~min}$, and pick-up time and delivery time of each fulcrum station as $40 \mathrm{~min}$. Set the maximum number of cars at each fulcrum station as $1300,800,1200,900$ in turn.The maximum number of marshalling cars in a train set to 55 . Suppose station $C$ stoped operation at 23:00 that day due to natural disasters. The train diagram was shown in figure 2. The initial car flows and their routings were shown in table 5, where the generation moment was the conversion minutes. The loading plan was shown in table 6 and the emptying plan was shown in table 7. 

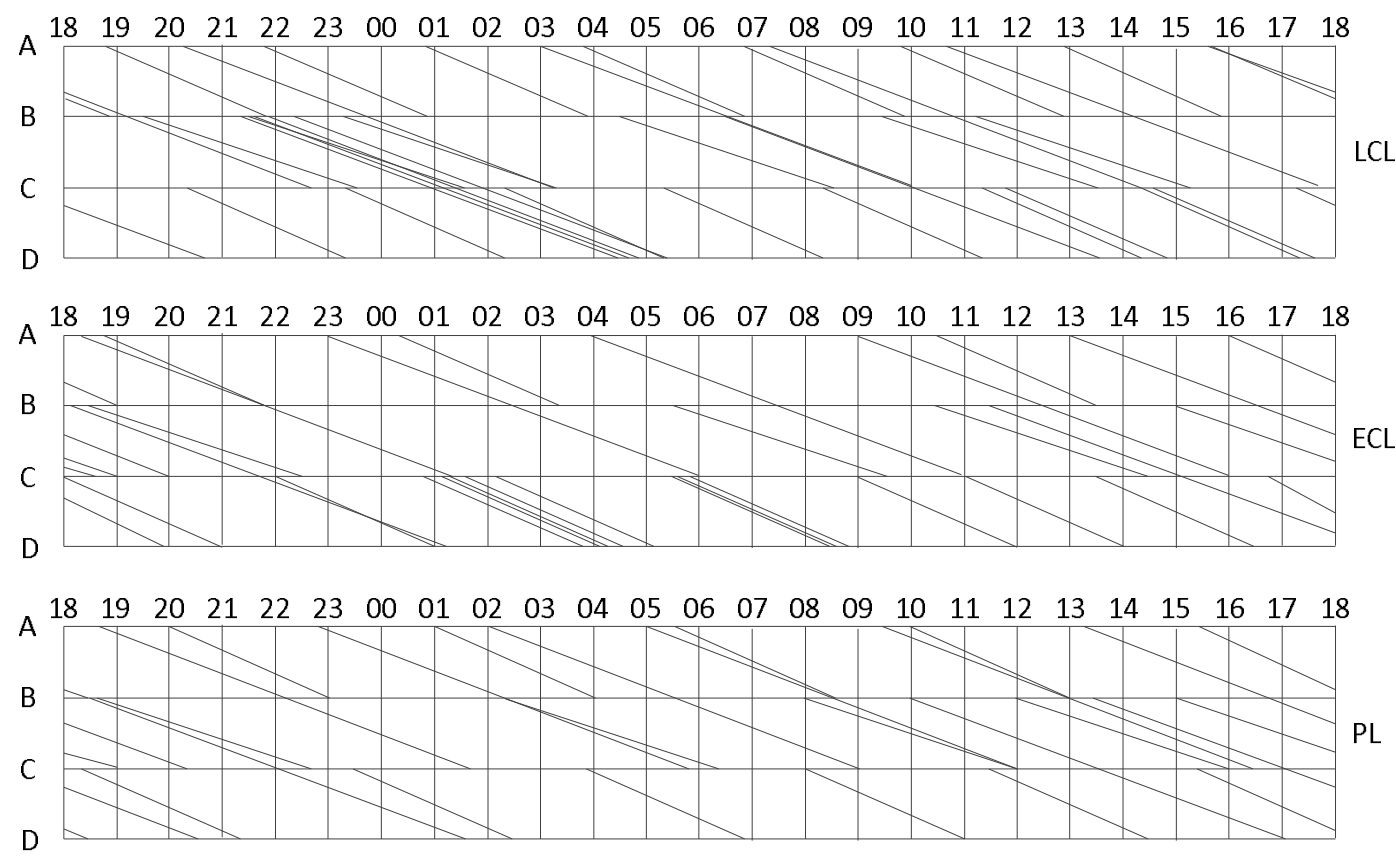

Figure 2. Train diagram

Table 5. Initial car flows and their routings

\begin{tabular}{ccccc}
\hline O & D & Car flow quantity(Generation moment) & Type & Car flow routing \\
\hline A & B & $100(0), 200(180), 150(250)$ & Loaded & A-B \\
A & C & $50(0), 60(120), 90(160)$ & Loaded & A-C \\
A & D & $30(0), 30(160), 40(200)$ & Loaded & A-C-D \\
B & C & $20(100), 50(300)$ & Loaded & B-C \\
B & D & $150(0)$ & Loaded & B-D \\
C & D & $60(0), 50(100), 80(160), 100(230), 50(350)$ & Loaded & C-D \\
A & $200(100), 20(170), 100(230), 80(310), 30(350)$ & Empty & \\
B & $60(0), 100(160), 60(200), 100(270), 60(360)$ & Empty & \\
C & $100(120), 80(250), 160(300), 150(360), 150(450)$ & Empty & \\
\hline
\end{tabular}

Table 6. Loading plan

\begin{tabular}{cccc}
\hline $\begin{array}{c}\text { Loading } \\
\text { station }\end{array}$ & $\begin{array}{c}\text { Destination } \\
\text { station }\end{array}$ & $\begin{array}{c}\text { Planned loading } \\
\text { quantity }\end{array}$ & $\begin{array}{c}\text { Completed loading } \\
\text { quantity }\end{array}$ \\
\hline A & B & 120 & 0 \\
A & C & 160 & 0 \\
B & C & 100 & 0 \\
B & D & 100 & 0 \\
C & D & 240 & 0 \\
\hline
\end{tabular}

Table 7. Emptying plan

\begin{tabular}{ccc}
\hline Boundary station & Planned emptying quantity & Completed emptying quantity \\
\hline $\mathrm{D}$ & 300 & 0 \\
\hline
\end{tabular}


C\# programming was adopted to realize the construction of space-time network, and 307 space-time nodes and 584 space-time arcs were generated. A feasible path set generation algorithm based on improved $\mathrm{A}^{*}$ algorithm was used to generate the feasible path set between any pair of OD demands. The generation results of feasible path sets between OD demands were shown in table 8.

Table 8. The generation results of feasible path sets

\begin{tabular}{ccccc}
\hline OD demand & IA quantity & SOP quantity & ETLP quantity & DP quantity \\
\hline A-B & 25 & 0 & 12 & 1 \\
A-C & 17 & 0 & 19 & 1 \\
A-D & 6 & 0 & 12 & 1 \\
B-C & 31 & 6 & 22 & 1 \\
B-D & 5 & 6 & 36 & 1 \\
C-D & 11 & 23 & 51 & 1 \\
\hline
\end{tabular}

Since this model was a linear programming model, the distribution results of car flow in the space-time network can be obtained by using the optimization solution software CPLEX. The loaded car flows were loaded onto the space-time arc, and the flow distribution results of some space-time arcs were shown in table 9. The car flow quantity of the TA numbered 2 and 36 in the table was 0 , indicating that some trains stop running after abnormal conditions occurred at station $\mathrm{C}$. According to the distribution results of space-time arc, the real-time quantity of vehicles stored at the station can be obtained. The ratio of the real-time quantity of cars to the maximum quantity of cars at the station was taken as the index to measure whether the station reached the bottleneck, that was, the station load rate(SLR). When SLR was greater than 1, the station was in the bottleneck state at that moment. SLR time series curves of each station were shown in figure 3. It can be concluded from the figure that station B was in the bottleneck state from 16:00 to 18:00 of the next day, and other stations were in the normal state.

Table 9. The car flow distribution results of partial space-time arcs

\begin{tabular}{cccccccc}
\hline Number & O & D & Type & Hierarchy & Car flow quantity & Start moment & End moment \\
\hline : & & & & & & & \\
2 & A & C & TA & LCL & 0 & 420 & 840 \\
5 & C & D & TA & LCL & 55 & 140 & 320 \\
36 & B & C & TA & ECL & 0 & 690 & 930 \\
60 & A & B & TA & PL & 55 & 960 & 1140 \\
108 & A & A & SA & LCL & 65 & 480 & 590 \\
241 & B & B & SOA & LCL & 55 & 1050 & 1230 \\
581 & C & C & DA & LCL & 280 & 0 & 1440 \\
\hline
\end{tabular}




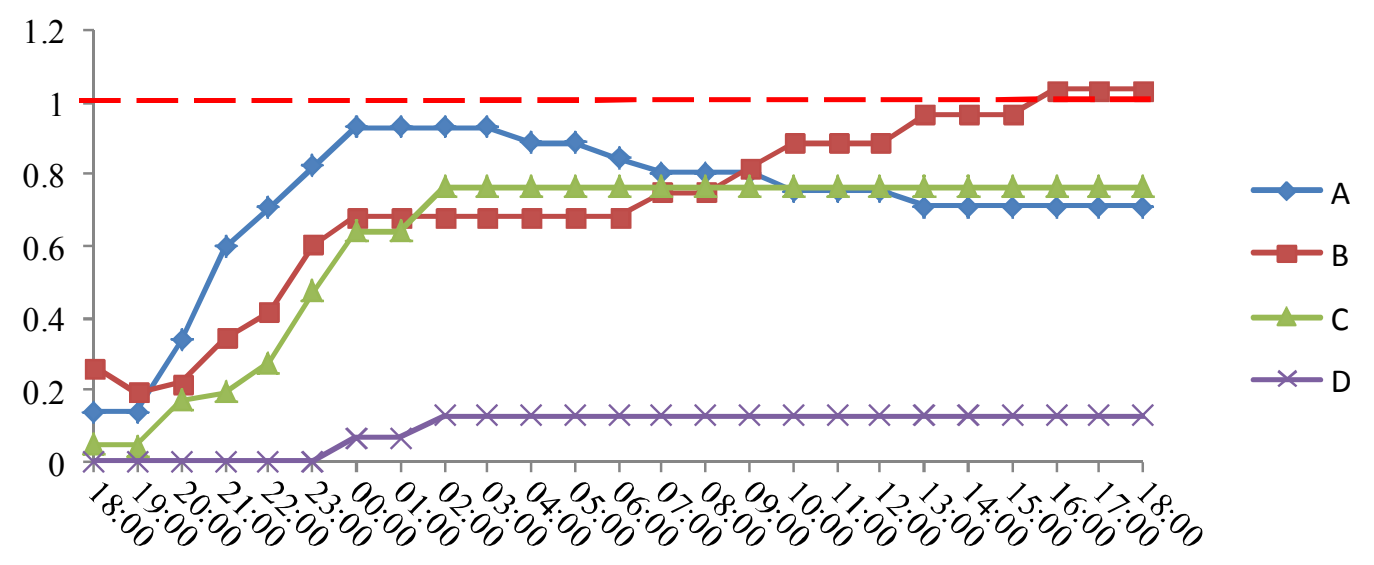

Figure 3. SLR time series curves corresponding to different stations

The solution of MDCFOONC was used to represent the dynamic car flow organization process under normal conditions, and it was compared with the solution of MDCFOOAC. Comparison of total loss(TL), comparison of average loading plan complete rate(ALPCR) and comparison of average emptying plan complete rate(AEPCR) under two different conditions was shown in figure 4 . As can be seen from the figure, when abnormal conditios occurred at station $C$ at 23:00, the TL of the transport system would increase by $16.6 \%$, the ALPCR would be reduced from $100 \%$ to $25 \%$, and the AEPCR would be reduced from $58 \%$ to $19 \%$.

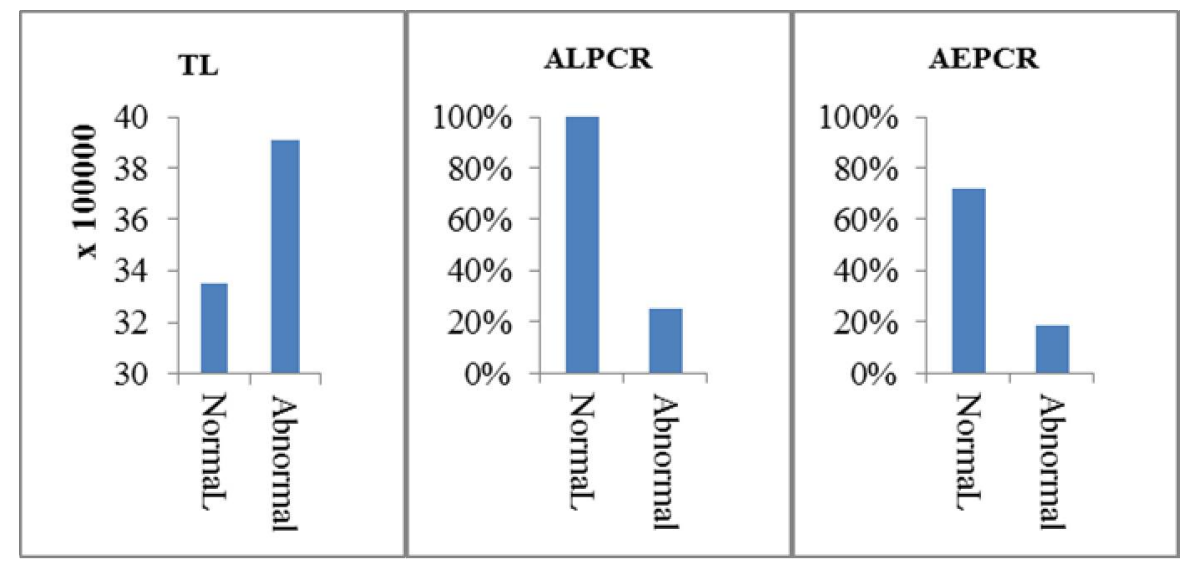

Figure 4. Index comparison

Aiming at the overstock phenomenon of car flow in station B, the restriction loading strategy can be adopted to solve the bottleneck of the station. In order to obtain the appropriate loading quantity, the loading quantity of the loading plan whose destination station was station B can be continuously reduced, and then SLR time series curves of station $\mathrm{B}$ can be obtained by using the solution of MDCFOOAC. In this paper, the gradient was set as 20 , and the loading quantity of the loading plan whose destination station was station B was set as 100, 80, 60, 40 and 20 successively. The SLR time series curves of station B corresponding to different loading quantity of the loading plan was shown in figure 5. In order to facilitate observation, the SLR time series curves from 13:00 to 18:00 of the next day at station B was mainly intercepted. As can be seen from the figure, when the loading quantity of the loading plan was constantly reduced, the real-time SLR of station B was constantly decreasing. When 
the loading quantity of the loading plan was 80, the real-time SLR of station B during this period was less than 1 , indicating that the dynamic bottleneck of station $B$ had been solved. Although the loading quantity of the loading plan could be reduced, and the SLR of station B will be lower, but the economic benefits of the transportation system will be reduced. Therefore, it is relatively reasonable to adjust the loading quantity of the loading plan to 80 . Of course, in order to get more ideal loading quantity of the loading plan, the gradient can be set to smaller.

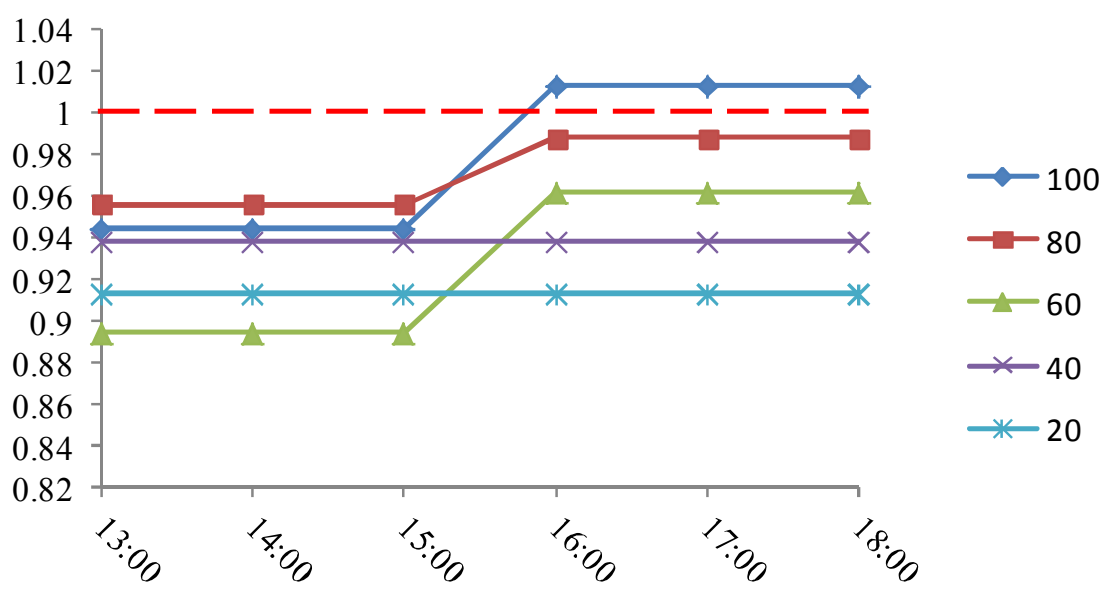

Figure 5. SLR time series curves of station B corresponding to different loading quantity

\section{Conclusion}

By combining the train diagram for space-time expansion, the railway physical network is transformed into a space-time network. The solution of dynamic traffic flow organization optimization model is used to simulate the field operation process. Based on the results of car flow distribution, the real-time quantity of cars in the station can be estimated, thus realizing the identification of dynamic bottleneck station. The information obtained by this method can provide decision-making basis for car flow adjustment of railway dispatching departments and is of great application value for reducing the overstock of car flow in railway stations.

\section{Acknowledgments}

This paper is supported by National Key R\&D Program of China (2018YFB1201402). The authors acknowledge the financial support of China Railway Corporation, the guidance of professor He.

\section{Author Contributions}

Methodology, Zihan Qu; Resources, Shiwei He; Data Curation, Zihan Qu; Writing - Original Draft Preparation, Zihan Qu; Writing - Review \& Editing, Zihan Qu; Project Administration, Shiwei He; Funding Acquisition, Zihan Qu.

\section{Reference}

1. Backfrieder C, Ostermayer G, Mecklenbrauker C F . Increased Traffic Flow Through Node-Based Bottleneck Prediction and V2X Communication[J]. IEEE Transactions on Intelligent Transportation Systems, 2016, 18(2):1-15.Zhang W, Lu J, Zhang Y. Comprehensive Evaluation Index System of Low Carbon Road Transport Based on Fuzzy Evaluation Method[J]. Procedia Engineering, 2016, 137:659-668. 
2. Ros B G, Knoop V L, Shiomi Y, et al. Modeling Traffic at Sags[J]. International Journal of Intelligent Transportation Systems Research, 2016, 14(1):64-74.

3. Lee W H , Tseng S S , Shieh J L , et al. Discovering Traffic Bottlenecks in an Urban Network by Spatiotemporal Data Mining on Location-Based Services[J]. IEEE Transactions on Intelligent Transportation Systems, 2011, 12(4):1047-1056.

4. Ma J , Li C , Liu Z, et al. On Traffic Bottleneck in Green ITS Navigation: An Identification Method[C]// IEEE Vehicular Technology Conference. IEEE, 2016.

5. Davis L C . Mitigation of congestion at a traffic bottleneck with diversion and lane restrictions[J]. Physica A Statistical Mechanics \& Its Applications, 2012, 391(4):1679-1691.

6. Qiu S, Luo W, Yao L, et al. A comparative study of funnel shape bottlenecks in subway stations[J]. Transportation Research Part A, 2017, 98:14-27.

7. $\mathrm{Xu} \mathrm{X} \mathrm{Y,} \mathrm{Liu} \mathrm{J,} \mathrm{Li} \mathrm{H} \mathrm{Y,} \mathrm{et} \mathrm{al.} \mathrm{Analysis} \mathrm{of} \mathrm{subway} \mathrm{station} \mathrm{capacity} \mathrm{with} \mathrm{the} \mathrm{use} \mathrm{of} \mathrm{queueing}$ theory[J]. Transportation Research Part C: Emerging Technologies, 2014, 38:28-43.

8. Ma J , Xu S M , Li T, et al. Method of Bottleneck Identification and Evaluation During Crowd Evacuation Process[J]. Procedia Engineering, 2014, 71:454-461.

9. Jie L, Shengxue H E, Haodong Z , et al. Optimization analysis for serial bottleneck system of urban rail transit station[J]. Journal of Computer Applications, 2016.

10. Jinhua S, Ying Z, Junmin C, et al. Analysis of Crowded Degree of Emergency Evacuation at "Bottleneck" Position in Subway Station Based on Stairway Level of Service[J]. Procedia Engineering, 2011, 11:242-251.

11. LIANG Dong,LIN Boliang. Research on the Model of Optimizing Strategically Dynamic Railcar Operation [J]. System Engineering Theory and Practice, 2007, 27(1):77-84.

12. GUO Pengwen,LIN Boliang. Section center optimization method for distribution of empty cars over large scale road network[J]. China Railway Science, 2001, 22(2):122-128.

13. TIAN Yaming, LIN Boliang, JI Lijun. Railway Car Flow Distribution Node-arc and Arc-path Models Based on Multi-commodity and Virtual Arc [J]. Journal of the China Railway Society, 2011, 33(4):7-12.

14. WANG Long, MA Jianjun, LIN Boliang, et al. Optimal Route Choice Model for Loaded and Empty Car Flows in Railway Network [J]. Journal of Beijing Jiaotong University, 2014, 38(6). 